\title{
Trapped Field Properties of GdBaCuO Bulk Superconductors of Various Diameters Magnetized by Pulsed Fields using an Identical Split Coil
}

\author{
Fumiya Shimoyashiki, Hiroyuki Fujishiro, Tomoyuki Naito, and Mark D. Ainslie, Senior Member, IEEE
}

\begin{abstract}
In this paper, the trapped field properties of $\mathrm{GdBaCuO}$ disk bulk superconductors of various diameters during pulsed-field magnetization (PFM) using an identical split coil at 65 $K$ have been investigated both experimentally and numerically. The maximum trapped field, $B_{\mathrm{T}}{ }^{\mathrm{max}}$, of the $\phi 43 \mathrm{~mm}$ bulk was larger than that of the $\phi 30 \mathrm{~mm}$ bulk. However, $B \mathrm{~T}^{\mathrm{max}}$ of the $\phi 65 \mathrm{~mm}$ bulk was smaller than that of the $\phi 43 \mathbf{~ m m}$ bulk and the trapped field profile exhibited a distorted " $C$-shaped" profile. Using the numerical simulation, these results for the $\phi 65 \mathrm{~mm}$ bulk can be explained by an inhomogeneous temperature profile and the larger generated heat, $Q$, due to the lower cooling power of the refrigerator compared to the generated heat. The important issues to achieve higher and homogeneous trapped fields are discussed when using splitcoil PFM for larger bulks.
\end{abstract}

Index Terms-Bulk superconductors, numerical simulation, pulsed-field magnetization, split coil, trapped field magnet.

\section{INTRODUCTION}

B ULK superconductors, acting as trapped field magnets (TFMs), can achieve higher magnetic fields of magnitude over ten times higher than the maximum field of conventional permanent magnets. The pulsed-field magnetization (PFM) is a practical magnetizing technique using a copper coil magnet, which is a clear contrast to field-cooled magnetization (FCM) using a superconducting coil magnet [1]. However, the trapped field, $B_{\mathrm{T}}$, by PFM is generally much smaller than that by FCM because of a large temperature rise due to the dynamical motion of magnetic flux [2]. To enhance the $B_{\mathrm{T}}$ value, multi-pulse techniques have been shown to be effective [3], [4], in which we have achieved a record-high $B_{\mathrm{T}}$ of $5.2 \mathrm{~T}$ at 30 $\mathrm{K}$ on a $\mathrm{GdBaCuO}$ bulk by using a modified multi-pulse technique combined with a stepwise cooling (MMPSC) using a solenoid coil [5].

This research is partially supported from JSPS KAKENHI Grant No. 15K04646. M. D. Ainslie would like to acknowledge financial support from an Engineering and Physical Sciences Research Council (EPSRC) Early Career Fellowship EP/P020313/1. All data are provided in full in the results section of this paper.

F. Shimoyashiki, H. Fujishiro, and T. Naito are with the Department of Physical Science and Materials Engineering, Faculty of Science and Engineering, Iwate University, Morioka 020-8551, Japan (e-mail: g0317083@iwate-u.ac.jp; fujishiro@iwate-u.ac.jp; tnaito@iwate-u.ac.jp).

M. D. Ainslie is with Bulk Superconductivity Group, Department of Engineering, University of Cambridge CB2 1PZ, UK (e-mail: mark.ainslie@eng.cam.ac.uk).
Based on Bean's critical-state model [6], the trapped field at the center of the disk bulk is proportional to the bulk diameter for an identical critical current density, $J_{c}$. The trapped field properties by PFM have been investigated for larger disk bulks $60 \mathrm{~mm}$ in diameter at $40 \mathrm{~K}$ using a solenoid coil [7], and 60 and $140 \mathrm{~mm}$ diameters at $77 \mathrm{~K}$ using a vortex coil [8], [9]. However, the $B_{\mathrm{T}}$ value was not necessarily enhanced, compared to that for smaller bulks $30-45 \mathrm{~mm}$ in diameter [5], [10]. This is because the $J_{\mathrm{c}}$ value of the larger bulks was not necessarily as large as that of the smaller bulks and the $J_{\mathrm{c}}$ distribution was inhomogeneous.

The use of vortex or split coils has also been shown to be effective to enhance the $B_{\mathrm{T}}$ value for smaller bulks during PFM, both experimentally and numerically [11], [12]. It is easier for the magnetic flux to penetrate via flux jumps, which can enhance the trapped field for REBaCuO bulks. We have previously reported the trapped field properties of a $30 \mathrm{~mm}$ diameter $\mathrm{GdBaCuO}$ bulk magnetized by a split coil, for which $B_{\mathrm{T}}=$ $3.2 \mathrm{~T}$ was achieved at $40 \mathrm{~K}$ using a single magnetic pulse of 5.0 T [13]. However, there has not yet been a systematic investigation of the trapped field properties by PFM using such a split coil for the disk bulks of various diameters at lower temperatures. The research of the large-sized TFMs and the enhancement of the trapped field using the split coil are valuable for engineering applications such as superconducting rotating machines, which requires large field poles.

In this paper, the trapped field properties of $\mathrm{GdBaCuO}$ bulk superconductors 43 and $65 \mathrm{~mm}$ in diameter using the split coil at $65 \mathrm{~K}$ are investigated experimentally and numerically, and compared with our previous data for the $30 \mathrm{~mm}$ diameter $\mathrm{GdBaCuO}$ bulk [14]. The possibility of trapped field enhancement for larger disk bulks using the split coil is discussed.

\section{EXPERIMENTAL AND NUMERICAL FRAMEWORK}

\section{A. Experimental PFM procedure}

$\mathrm{GdBaCuO}$ disk bulks of various diameters $(\phi 43 \mathrm{~mm} \times H 18$ $\mathrm{mm}$, and $\phi 65 \mathrm{~mm} \times H 20 \mathrm{~mm}$; Nippon Steel \& Sumitomo Metal) were magnetized using a split coil (72 $\mathrm{mm}$ in inner diameter (I.D.), $124 \mathrm{~mm}$ in outer diameter (O.D.) and $37 \mathrm{~mm}$ in height $(\mathrm{H})$ ), in which a set of permendur yokes $(60 \mathrm{~mm}$ in di- 
ameter and $65 \mathrm{~mm}$ in height) was inserted. Each disk bulk was fastened in brass sample holders of different sizes using a thin indium sheet and was thermally connected to the cold stage of GM-cycle He refrigerator (5 W at $20 \mathrm{~K}$ ), as shown in Fig. 1, in which the bulk was cooled from the periphery along the $a b$ plane of the bulk. The detailed experimental setup for PFM is described in [14]. The $65 \mathrm{~mm}$ bulk was also magnetized using a solenoid coil $(95 \mathrm{~mm}$ in I.D., $121 \mathrm{~mm}$ in O.D. and $50 \mathrm{~mm}$ in $\mathrm{H})$ for comparison. The results are then compared to our previous PFM results using the same split coil for a $\mathrm{GdBaCuO}$ disk bulk $(\phi 30 \mathrm{~mm} \times H 14 \mathrm{~mm})$ fabricated by the University of Cambridge [14]. A single magnetic pulse, $B_{\mathrm{ex}}$, with a rise time of $18 \mathrm{~ms}$ was applied, ranging from 2.7 to $6.0 \mathrm{~T}$ at $T_{\mathrm{s}}=65 \mathrm{~K}$. The results at $65 \mathrm{~K}$ can clearly compare with the numerical ones. The time evolution of the magnetic field, $B_{\mathrm{T}}(t)$, and the final trapped field, $B_{\mathrm{T}}$, were measured at the center of bulk surface by a Hall sensor (F. W. Bell, BHT921) (adhered to the surface), and a two-dimensional (2D) trapped field profile was measured at a distance of $3 \mathrm{~mm}$ above the bulk surface, stepwise with a pitch of $1 \mathrm{~mm}$, by scanning an axial-type Hall sensor (F. W. Bell, BHA921). The time evolution of the temperature, $T(t)$, was also measured using the Cernox $^{\mathrm{TM}}$ thermometer connected to the sample holder as shown in Fig. 1. A heater was used to control the operating temperature using a Pt-Co thermometer.

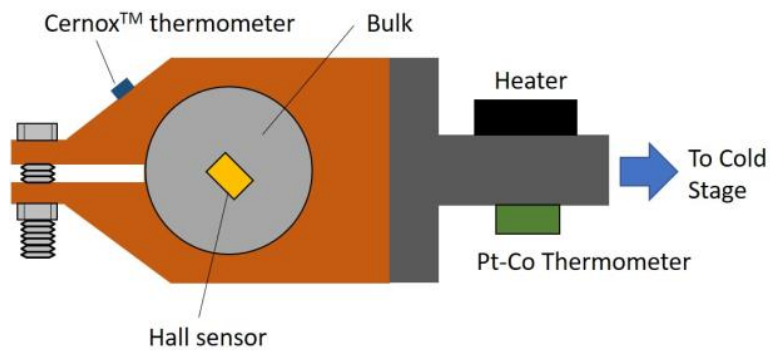

Fig. 1. A schematic view of the disk bulk and brass sample holder, which is thermally connected to the cold stage of the refrigerator.

\section{B. Numerical simulation framework}

Based on our experimental setup [14], a three dimensional (3D) numerical model was constructed using the finite element method (FEM). The commercial software package, Photo-Eddy, combined with Photo-Thermo (Photon Ltd, Japan), was used for the analysis. The following magnetic field dependence of $J_{\mathrm{c}}(B)$, proposed by Jirsa, was used in the simulation [15],

$$
J_{c}(B)=J_{c 1} \exp \left(-\frac{B}{B_{L}}\right)+J_{c 2} \frac{B}{B_{\max }} \exp \left[\frac{1}{k}\left(1-\left(\frac{B}{B_{\max }}\right)^{k}\right)\right] .
$$

The experimental $J_{\mathrm{c}}(B, T)$ data [16] were fit up to $10 \mathrm{~T}$ between $65,70,75$ and $80 \mathrm{~K}$ using eq. (1) and the parameters $\left(J_{\mathrm{c} 1}, B_{\mathrm{L}}, J_{\mathrm{c} 2}, B_{\max }\right.$ and $\left.k\right)$ were determined at each temperature. For example, each parameter value at $65 \mathrm{~K}$ was $J_{\mathrm{cl}}=1.17 \times$ $10^{9} \mathrm{~A} / \mathrm{m}^{2}, B_{\mathrm{L}}=0.57 \mathrm{~T}, J_{\mathrm{c} 2}=7.56 \times 10^{8} \mathrm{~A} / \mathrm{m}^{2}, B_{\max }=3.0 \mathrm{~T}$ and $k=1.3$. The $J_{\mathrm{c}}(B, T)$ profiles at intermediate magnetic fields and temperatures are interpolated based on each parameter [17].

\section{RESULTS AND DISCUSSION}

\section{A. Experimental results}

Fig. 2(a) shows the trapped field, $B_{\mathrm{T}}$, at $65 \mathrm{~K}$ at the center of the surface of the $\phi 65 \mathrm{~mm}$ bulk, as a function of the applied field, $B_{\text {ex }}$, using the split and solenoid coils. The typical trapped field profiles are also shown in Figs. 2(b) - 2(e). For both coils, $B_{\mathrm{T}}$ increases with increasing $B_{\mathrm{ex}}$, takes a maximum, and then decreases. The maximum trapped field, $B_{\mathrm{T}}{ }^{\max }$, was $1.73 \mathrm{~T}$ for the solenoid coil and $1.57 \mathrm{~T}$ for the split coil without (w/o) the permendur yoke. However, $B_{\mathrm{T}}{ }^{\max }$ was enhanced to $2.24 \mathrm{~T}$ by about $29 \%$ by inserting the magnetic yoke into the bore of the split coil. For the split coil, a higher $B_{\mathrm{T}}{ }^{\max }$ can be achieved for lower $B_{\text {ex }}$ than for the solenoid coil. For lower $B_{\text {ex }}$, as shown in Fig. 2(b) for the split coil and Fig. 2(c) for the solenoid coil, the trapped field profiles were homogenous. For higher $B_{\text {ex }}$ using the solenoid coil, as shown in Fig. 2(d), the trapped field profile exhibited a conical shape, which suggests that the $J_{\mathrm{c}}$ distribution is homogeneous in the bulk. However, for higher $B_{\text {ex }}$, as shown in Fig. 2(e) for the split coil, the trapped field profile was fairly distorted and exhibited a "C-shaped profile". The similar distorted profile to Fig. 2(e) was also observed for the bulk with yoke. However, it is hypothesized that the distorted profile does not come from an inhomogeneous $J_{\mathrm{c}}$ distribution, but rather that the temperature distribution within the bulk is inhomogeneous during PFM in the case of the split coil at higher $B_{\mathrm{ex}}$, for which a large amount of heat is generated in the bulk due to pinning loss and viscous loss, if the cooling power of the refrigerator is smaller than the generated heat. A large amount of heat generated in the region opposite the cooling side is difficult to be extracted out to the cold stage.

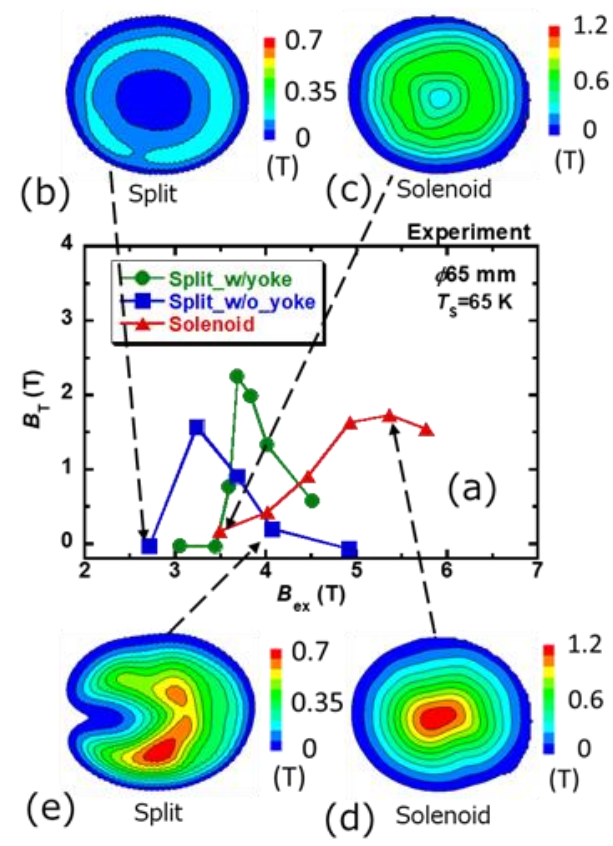

Fig. 2. (a) The trapped field, $B_{\mathrm{T}}$, at the center of the bulk surface, as a function of the applied field, $B_{\mathrm{ex}}$, at $T_{\mathrm{s}}=65 \mathrm{~K}$ for the solenoid and split coils. The typical trapped field profiles are shown in $(b) \sim(e)$ for selected points from (a).

As a result, the temperature rise in that region causes a localized decrease in $J_{\mathrm{c}}$, and then the trapped flux leaves the bulk 
easily. On the other hand, for the solenoid coil, the bulk was cooled along $c$-axis through a large area, and the temperature in the $a b$-plane of the bulk is relatively homogeneous. The detail discussion is performed later with the aid of numerical simulations.

Fig. 3 shows the trapped field, $B_{\mathrm{T}}$, at the center of the bulk surface for the various diameters, as a function of the applied field, $B_{\text {ex }}$, at $65 \mathrm{~K}$ using a split coil with the permendur yoke. For all bulks, $B_{\mathrm{T}}$ increases, takes a maximum and then decreases with increasing $B_{\text {ex }}$. The $B_{\mathrm{T}}{ }^{\max }$ of the $\phi 43 \mathrm{~mm}$ bulk was larger than that of the $\phi 30 \mathrm{~mm}$ bulk. However, $B_{\mathrm{T}}{ }^{\max }$ of the $\phi 65 \mathrm{~mm}$ bulk was lower than that of the $\phi 43 \mathrm{~mm}$ bulk, and $B_{\mathrm{T}}$ abruptly increased and then decreased, compared to the other bulks. If the $J_{\mathrm{c}}(B, T)$ characteristics of the bulks are assumed to be identical and homogeneous, the magnetic flux starts to penetrate into the bulk center at a higher $B_{\text {ex }}$, and the larger disk bulk should trap a higher magnetic field at the higher $B_{\text {ex }}$. The experimental results for the $65 \mathrm{~mm}$ bulk suggest that an inhomogeneous $J_{\mathrm{c}}$ distribution (due to the large heat generation) exists in the bulk and, as a result, the magnetic flux easily penetrates at lower $B_{\mathrm{ex}}$ and easily escapes at higher $B_{\text {ex }}$. The maximum $B_{\mathrm{T}}{ }^{\text {max }}$ among all the bulks was 2.34 $\mathrm{T}$ for the $\phi 43 \mathrm{~mm}$ bulk. This discrepancy mainly comes from the inhomogeneous $J_{\mathrm{c}}$ distribution in the $65 \mathrm{~mm}$ bulk due to the inhomogeneous heat transfer to the sample holder and cold stage.

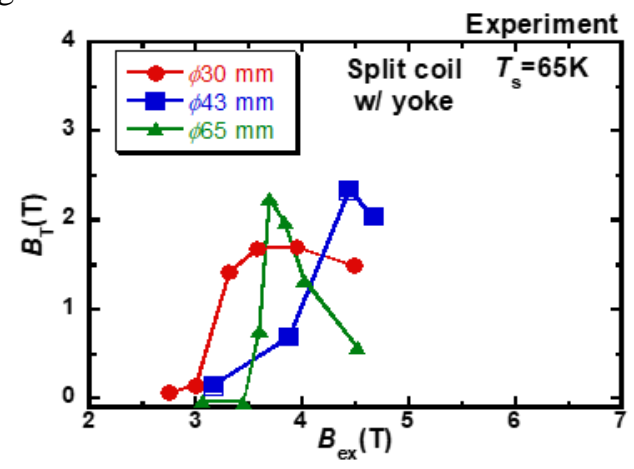

Fig. 3. The trapped field, $B_{\mathrm{T}}$, at the center of the bulk surface for the bulks of various diameters magnetized by a split coil with yoke at $65 \mathrm{~K}$, as a function of the applied field, $B_{\text {ex }}$.

Fig. 4(a) shows the maximum temperature rise, $\Delta T_{\max }$, as a function of the applied field, $B_{\mathrm{ex}}$, for the bulks with various diameters, which was measured on the bulk holder. $\Delta T_{\max }$ increases with increasing $B_{\text {ex }}$ for each bulk and the gradient of $\Delta T_{\max } / B_{\text {ex }}$ becomes steeper with increasing diameter of the bulk. Fig. 4(b) shows the $B_{\text {ex }}$ dependence of the generated heat, $Q$, for each bulk, which was estimated using following equation [7].

$$
Q=\int_{T_{S}}^{T_{S}+\Delta T_{\max }} C(T) V d T,
$$

where, $C(T)$ is specific heat per unit volume and $V$ is the volume of the $\mathrm{GdBaCuO}$ bulk [14]. The $Q$ value in Fig. 4(b) shows a similar $B_{\text {ex }}$ dependence to $\Delta T_{\max }$ shown in Fig. 4(a), which results from the pinning loss due to flux pinning and the viscous loss due to flux movement, but also changes depending on the $J_{\mathrm{c}}(B, T)$ value. The $Q$ values of the $\phi 65 \mathrm{~mm}$ bulk indicate larger values, compared to those of the other bulks. Since $V=66.3 \mathrm{~cm}^{3}$ for the $\phi 65 \mathrm{~mm}$ bulk, $26.1 \mathrm{~cm}^{3}$ for the $\phi 43 \mathrm{~mm}$ bulk, and $10.6 \mathrm{~cm}^{3}$ for the $\phi 30 \mathrm{~mm}$ bulk, the $Q$ values are nearly proportional to the $V$ value more than $B_{\mathrm{ex}}=$ $3.5 \mathrm{~T}$. It should be noted that $J_{\mathrm{c}}(B, T)$ characteristics of the bulks used in the experiment have not been measured/estimated and may be different to each other. The cooling power of the refrigerator used in this study was $5 \mathrm{~W}(=5$ $\mathrm{J} / \mathrm{s}$ ) at $20 \mathrm{~K}$, which is quite smaller than the generated heat during PFM. The brass holder for each bulk has different size and heat capacity, which also influences the temperature rise and the heat propagation. As a result, the temperature of the bulk increases due to the generated heat exceeding the cooling ability, especially for the larger bulk.
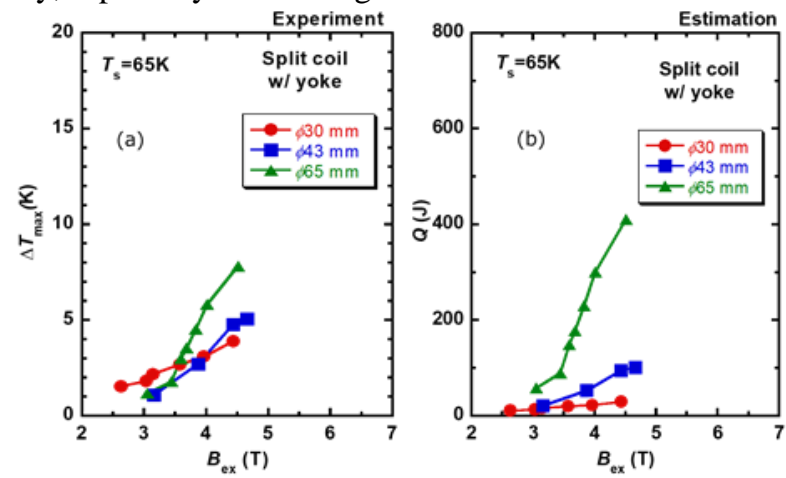

Fig. 4 (a) The maximum temperature rise, $\Delta T_{\max }$, of the sample holder of the bulks of various diameters. (b) The generated heat, $Q$, as a function of the applied field, $B_{\mathrm{ex}}$, which was estimated using eq. (2).

\section{B. Numerical simulation results}

In the numerical simulation, the $J_{\mathrm{c}}(B, T)$ characteristics are assumed to be identical and homogeneous for all the bulks. Fig. 5 shows the trapped field, $B_{\mathrm{T}}$, at the center of the bulk surface without yoke, as a function of the applied field, $B_{\mathrm{ex}}$. In all cases, $B_{\mathrm{T}}$ increased with increasing $B_{\mathrm{ex}}$, took a broad peak and then decreased. It is easier for the magnetic flux to penetrate to the center of the bulk (and be subsequently trapped) for a smaller diameter sample. The $B_{\mathrm{T}}{ }^{\max }$ value increases with increasing bulk diameter, which does not reproduce the experimental results shown in Fig. 3. The $B_{\text {ex }}$ value to achieve $B_{\mathrm{T}}{ }^{\max }$ increases with increasing bulk diameter. These results suggest that it is harder for the magnetic flux penetration into the center of the larger bulk. The sudden increase and steep decrease in $B_{\mathrm{T}}$ for the $65 \mathrm{~mm}$ bulk shown in Fig. 3 was not reproduced by the simulation under these initial assumptions.

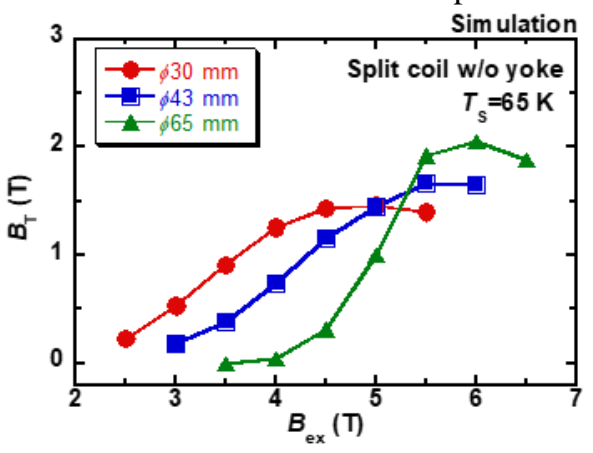

Fig. 5. Numerical simulation results of the trapped field, $B_{\mathrm{T}}$, at the center of the bulk surface at $65 \mathrm{~K}$ using the split coil without yoke, as a function of the applied field, $B_{\mathrm{ex}}$, for the bulks of various diameters. 
Fig. 6(a) shows the maximum temperature rise, $\Delta T_{\max }$, as a function of the applied field, $B_{\mathrm{ex}}$, for the bulks of various diameters, which was estimated at the same position as the experiment in Fig. 1. $\Delta T_{\max }$ increases with increasing $B_{\text {ex }}$ for each bulk. However, the magnitude of $\Delta T_{\max }$ is larger than that of experimental results. Furthermore, the gradient of $\Delta T_{\max } / B_{\text {ex }}$ is nearly identical, which is quite different to the experimental results.

Fig. 6(b) shows the $B_{\text {ex }}$ dependence of the generated heat, $Q$, for each bulk, which was estimated using eq. (2). The results show a similar trend as the experimental results in Fig. 3(b). However, the maximum $Q$ value for the $\phi 65 \mathrm{~mm}$ bulk is about twice as large as that measured in the experiments. In the actual bulks, there exist regions of lower $J_{\mathrm{c}}$, through which the magnetic flux penetrates easily, even at lower $B_{\text {ex }}$. As a result, the generated heat becomes small. The "C-shaped" trapped field profile in Fig. 2(e) suggests the possibility of an inhomogeneous temperature distribution in the large bulk due to the insufficient cooling power of the refrigerator. The numerical simulation was performed assuming an inhomogeneous initial temperature distribution in the bulk.
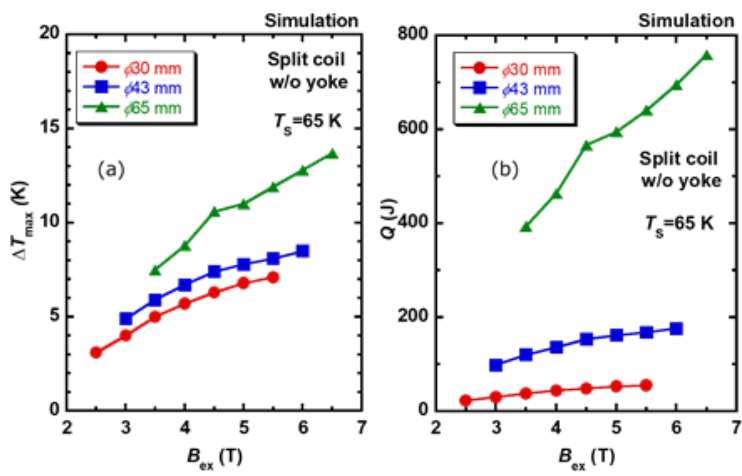

Fig. 6. Numerical simulation results of (a) maximum temperature rise, $\Delta T_{\max }$, and (b) generated heat, $Q$, as a function of the applied field, $B_{\text {ex }}$, for the bulks of various diameters.

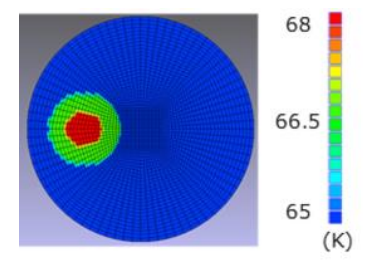

Fig. 7. Initial temperature distribution of the $\phi 65 \mathrm{~mm}$ bulk, in which regions 1.5 and $3 \mathrm{~K}$ higher than the rest of the bulk $(=65 \mathrm{~K})$ were assumed.

Fig. 7 shows the initial temperature distribution for the $\phi 65$ $\mathrm{mm}$ bulk, in which the regions 1.5 and $3 \mathrm{~K}$ higher than the rest of the bulk $(=65 \mathrm{~K})$ were assumed on the offside opposite to the cold stage. Fig. 8(a) shows the trapped field, $B_{\mathrm{T}}$, at the center of the bulk surface, as a function of $B_{\mathrm{ex}}$ for the $\phi 65 \mathrm{~mm}$ bulk in the case of the initial inhomogeneous temperature distribution shown in Fig. 7. The similar relationship for the bulk under the uniform $T_{\mathrm{s}}$ is also shown. The $B_{\mathrm{T}}$ vs $B_{\mathrm{ex}}$ curve for the inhomogeneous $T_{\mathrm{s}}$ distribution shifted to the lower $B_{\mathrm{ex}}$ side, and $B_{\mathrm{T}}{ }^{\text {max }}$ was decreased, compared to the uniform $T_{\mathrm{s}}$ case. The typical trapped field profiles are also shown in Figs. 8(b) - 8(e). In Figs. 8(c) and 8(d), for the inhomogeneous $T_{\mathrm{s}}$ distribution, the "C-shaped" trapped field profile can be reproduced at low and high $B_{\text {ex }}$. On the other hand, for the uniform $T_{\mathrm{s}}$ case, homogeneous trapped field profiles are observed, as shown in Figs. 8(b) and 8(e). These results indicated that the inhomogeneous $T_{\text {s }}$ profile can reproduce the "C-shaped" trapped field profile.

Finally, we should comment on the mechanical reinforcement required to withstand the large repulsion force during the ascending stage of PFM. The repulsion force increases with increasing the diameter of the bulk and with decreasing temperature due to the increase in the $J_{\mathrm{c}}$ value. Sufficient mechanical support is necessary to avoid damage to the refrigerator and/or the PFM apparatus.

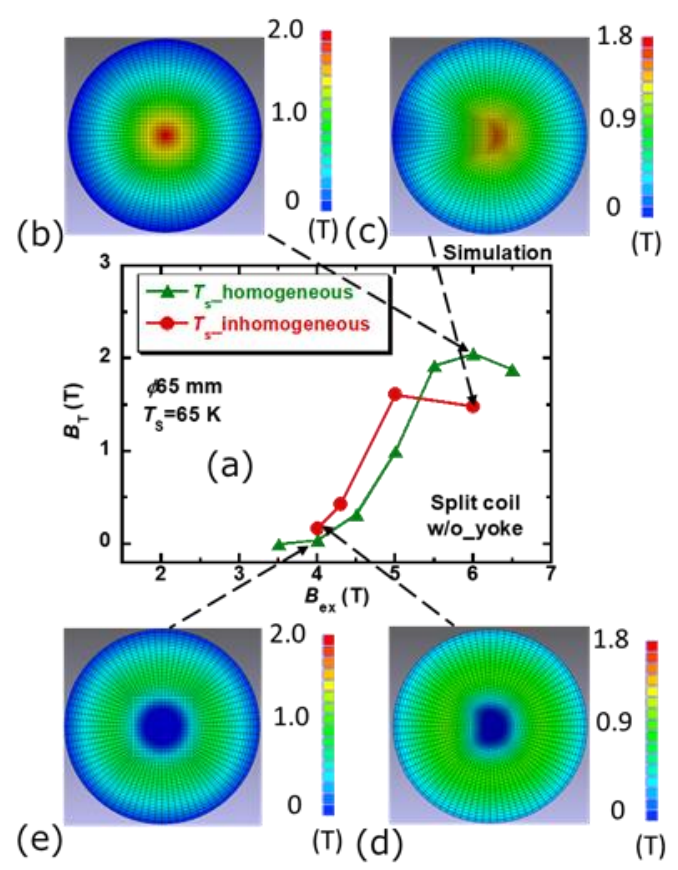

Fig. 8. (a) The trapped field, $B_{\mathrm{T}}$, at the center of the bulk surface, as a function of the $B_{\text {ex }}$ for the $\phi 65 \mathrm{~mm}$ bulk assuming the initial inhomogeneous temperature distribution shown in Fig. 7 and (b)-(e) typical trapped field profiles.

\section{CONCLUSION}

We have investigated the trapped field properties of $\mathrm{GdBaCuO}$ bulks with various diameters at $65 \mathrm{~K}$ during PFM using the split coil. The maximum trapped field, $B_{\mathrm{T}}{ }^{\max }$, of the $\phi 43 \mathrm{~mm}$ bulk was larger than that of the $\phi 30 \mathrm{~mm}$ bulk. However, $B_{\mathrm{T}}{ }^{\mathrm{max}}$ of the $\phi 65 \mathrm{~mm}$ bulk was smaller than that of the $\phi 43 \mathrm{~mm}$ bulk and the trapped field profile showed distorted "C-shaped" one. Using the numerical simulation, these results can be explained by the inhomogeneous temperature profile and by the larger generated heat, $Q$, for the $\phi 65 \mathrm{~mm}$ bulk due to the lower cooling power of the refrigerator than the generated heat. To achieve the higher and homogeneous trapped field profile for the larger bulk for PFM using the split coil, it is necessary to consider the cooling method and cooling power of the used refrigerator. 


\section{REFERENCES}

[1] J. H. Durrell, A. R. Dennis, J. Jaroszynski, M. D. Ainslie, K. G. B. Palmer, Y-H. Shi, A. M. Campbell, J. Hull, M. Strasik, E. E. Hellstrom and D. A. Cardwell, "A trapped field of 17.6 T in melt-processed, bulk Gd-Ba-Cu-O reinforced with shrink-fit steel”, Supercond. Sci. Technol. vol. 27, 2014, Art. no. 082001.

[2] A. Terasaki, Y. Yanagi, Y. Itoh, M. Yoshikawa, T. Oka, H. Ikuta, and U. Mizutani, "Flux Motion during Pulsed-Field Magnetization in MeltProcessed YBCO", Advances in Superconductivity X, Springer-Verlag, Tokyo 1998 pp. 945-948.

[3] Y. Yanagi, Y. Itoh, M. Yoshikawa, T. Pka, T. Hosokawa, H. Ishihara, H. Ikuta and U. Mizutani," Trapped Field Distribution on Sm-Ba-Cu-O Bulk Superconductor by Pulsed-Field Magnetization", Advances in Superconductivity XII, Springer-Verlag, Tokyo pp. 470-472.

[4] M. Sander, U. Sutter, R. Koch and M. Klaser, "Pulsed magnetization of HTS bulk parts at $T<77 \mathrm{~K}$ ", Supercond. Sci. Technol., vol. 13, 2000, pp. 841-845,

[5] H. Fujishiro, T. Tateiwa, A. Fujiwara, T. Oka and H. Hayashi, "Higher trapped field over 5 Tesla on HTSC bulk by modified pulse field magnetizing", Physica C, vol. 445-448, 2006, pp. 334-338.

[6] C. P. Bean, "Magnetization of hard superconductors", Phys. Rev. Lett. vol. 8, 1962, pp. 250-253.

[7] H. Fujishiro, T. Tateiwa, K. Kakehata, T. Hiyama and T. Naito, "Trapped Field and Temperature Rise on $\phi 65 \mathrm{~mm}$ GdBaCuO Bulk by Pulse Field Magnetization”, Supercond. Sci. Technol. vol. 20, 2007, pp. 1009-1014.

[8] E. Morita, H. Matsuzaki, Y. Kimura, H. Ogata, M. Izumi, T. Ida, M. Murakami, H. Sugimoto and M. Miki, "Study of a new split-type magnetizing coil and pulsed field magnetization of $\mathrm{Gd}-\mathrm{Ba}-\mathrm{Cu}-\mathrm{O}$ hightemperature superconducting bulk for rotating machinery application" Supercond. Sci. Technol. vol. 19, 2006, pp. 1259-1263.

[9] K. Yamaguchi, Y. Kimura, M. Izumi, S. Nariki, N. Sakai and I. Hirabayashi, "Pulsed Field Magnetization Properties for a Large Single grain Gd-Ba-Cu-O High-Temperature Superconductor Bulk with a Diameter of $140 \mathrm{~mm}$ by using a New Type of Pulsed Copper Split Coil" J. Phys.: Conf. Series vol. 97, 2008, Art. no. 012278

[10] M. D. Ainslie, D. Zhou, H. Fujishiro, K. Takahashi, Y-H. Shi and J. H. Durrell, "Flux jump-assisted pulsed field magnetization of high- $J_{\mathrm{c}}$ bulk high-temperature superconductors", Supercond. Sci. Technol. vol. 29, 2016, Art. no. 124004.

[11] T. Ida, H. Matsuzaki, Y. Akita, M. Izumi, H. Sugimoto, Y. Hondou, Y. Kimura, N. Sakai, S. Nariki, I. Hirabayashi, M. Miki, M. Murakami and M. Kitano, "Magnetization properties for $\mathrm{GdBaCuO}$ bulk superconductors with a couple of pulsed field vortex-type coils" Physica C. vol. 412414, 2004, pp. 638-645.

[12] M. D. Ainslie, H. Fujishiro, "Modelling of bulk superconductor magnetization", Supercond. Sci. Technol. vol. 28, 2015, Art. no. 053002.

[13] M. D. Ainslie, H. Fujishiro, H. Mochizuki, K. Takahashi, Y-H Shi, D. K. Namburi, J. Zou, D. Zhou, A. R. Dennis and D. A. Cardwell, "Enhanced trapped field performance of bulk high-temperature superconductors using split coil, pulsed field magnetization with an iron yoke" Supercond. Sci. Technol. vol. 29, 2016, Art. no. 074003

[14] K. Takahashi, M. D. Ainslie, H. Fujishiro, T. Naito, Y-H. Shi and D. A. Cardwell, "Trapped field properties of a $\mathrm{Y}-\mathrm{Ba}-\mathrm{Cu}-\mathrm{O}$ bulk by pulsed field magnetization using a split coil inserted by iron yokes with various geometries and electromagnetic properties", Physica C vol. 536, 2017, pp. 1-10.

[15] M. Jirsa, L. P̊ust, D. Dlouhý and M. R. Koblischka, "Fishtail shape in the magnetic hysteresis loop for superconductors: Interplay between different pinning mechanisms", Phys. Rev. B, vol. 55, 1997, pp. 3276-3284.

[16] T. Kii, R. Kinjo, N. Kimura, M. Shibata, M. A. Bakr, Y. W. Choi, M. Omer, K. Yoshida, K. Ishida, T. K. Komai, K. Shimahashi, T. Sonobe, H. Zen, K. Masuda and H. Ohgaki, "Low-Temperature Operation of a Bulk HTSC Staggered Array Undulator", IEEE Trans. Appl. Supercond. vol. 22, no. 3, 2012, Art. no. 4100904.

[17] T. Hirano, K. Takahashi, F. Shimoyashiki, H. Fujishiro, T. Naito, and M. D. Ainslie, "Influence of $J_{\mathrm{c}}(B, T)$ Characteristics on the Pulsed Field Magnetization of REBaCuO Disk Bulks" IEEE Trans. Appl. Supercond, to be published in IEEE Trans. Appl. Supercond. 2018. 